\title{
Testing and tuning symplectic integrators for the hybrid Monte Carlo algorithm in lattice QCD
}

\author{
Tetsuya Takaishi ${ }^{1}$ and Philippe de Forcrand ${ }^{2,3}$ \\ ${ }^{1}$ Hiroshima University of Economics, Hiroshima 731-0124, Japan \\ ${ }^{2}$ Physics Department, CERN, TH Unit, CH-1211 Genève 23, Switzerland \\ ${ }^{3}$ Institute for Theoretical Physics, ETH Zürich, CH-8093 Zürich, Switzerland \\ (Received 4 July 2005; revised manuscript received 18 November 2005; published 17 March 2006)
}

\begin{abstract}
We examine a new second-order integrator recently found by Omelyan et al. The integration error of the new integrator measured in the root mean square of the energy difference, $\left\langle\Delta H^{2}\right\rangle^{1 / 2}$, is about 10 times smaller than that of the standard second-order leapfrog (2LF) integrator. As a result, the step size of the new integrator can be made about three times larger. Taking into account a factor 2 increase in cost, the new integrator is about $50 \%$ more efficient than the $2 \mathrm{LF}$ integrator. Integrating over positions first, then momenta, is slightly more advantageous than the reverse. Further parameter tuning is possible. We find that the optimal parameter for the new integrator is slightly different from the value obtained by Omelyan et al., and depends on the simulation parameters. This integrator could also be advantageous for the Trotter-Suzuki decomposition in quantum Monte Carlo.

DOI: 10.1103/PhysRevE.73.036706

PACS number(s): 02.70.-c, 02.60.Cb, 11.15.Ha, 12.38.Gc
\end{abstract}

\section{INTRODUCTION}

The hybrid Monte Carlo (HMC) algorithm [1] is now the established standard for the generation of dynamical fermion configurations in lattice quantum chromodynamics (QCD). The HMC algorithm consists of molecular dynamics (MD) trajectories, each followed by a Metropolis test. During the MD trajectory, one integrates Hamilton's equations of motion, using an integrator with a discrete stepsize $\Delta t$ which must satisfy two conditions in order to maintain detailed balance: (i) simplecticity (the phase space volume $d p d q$ must be conserved) and (ii) time reversibility. The simplest and most widely used integrator with these properties is the secondorder leapfrog (2LF) integrator, which causes $\mathcal{O}\left(\Delta t^{2}\right)$ errors in the total energy or Hamiltonian. These errors are eliminated at the Metropolis accept/reject step, which makes the algorithm exact.

The acceptance at the Metropolis step depends on the magnitude of the error in the total energy. In order to reduce the error and thus increase the acceptance one could use higher order integrators. Early attempts, however, did not appear to be practical $[2,3]$. This is because the efficiency of higher order integrators depends largely on the system size and these early attempts were made on rather small lattices. As the lattice size increases above a certain value $V_{c}$, the higher order integrators should perform better than the low order integrator. This minimum lattice size $V_{c}$ depends on the Hamiltonian which we consider and on the choice of integrator. For lattice QCD, it turned out that $V_{c}$ becomes very large at small quark masses, so that on currently accessible computers the 2LF integrator is the best choice [4] for simulations at zero temperature. At finite temperature, higher order integrators could perform better on moderate-size lattices [5]: this is because chiral symmetry gets restored, so that small Dirac eigenvalues disappear, which allows for stable MD integration using larger stepsizes.

So far, only the $2 \mathrm{LF}$ integrator has been considered in the HMC algorithm of lattice QCD as a second-order integrator, because of its simplicity and effectiveness. Recently however, Omelyan et al. [6] found a new second-order integrator which is expected to be better than the 2LF integrator although it has twice the computational cost. Here we examine this new second-order integrator for the HMC algorithm in lattice QCD and measure its efficiency. We also examine the new fourth-order integrators recommended in Ref. [6]. Finally, we try to further tune the new second-order integrator.

\section{SYMPLECTIC INTEGRATOR}

\section{A. Recursive construction scheme}

Symplectic integrators are most conveniently described by the Lie algebra formalism [7-9]. Let $H$ be a classical Hamiltonian,

$$
H=\frac{1}{2} p^{2}+S(q),
$$

where $q=\left(q_{1}, q_{2}, \ldots\right)$ and $p=\left(p_{1}, p_{2}, \ldots\right)$ are coordinate variables and conjugate momenta, respectively. Hamilton's equations are expressed as

$$
\dot{f}=\{f, H\},
$$

where $f=q$ or $p$ and $\{$,$\} stands for the Poisson bracket,$

$$
\{f, H\}=\sum_{i}\left(\frac{\partial f}{\partial q_{i}} \frac{\partial H}{\partial p_{i}}-\frac{\partial f}{\partial p_{i}} \frac{\partial H}{\partial q_{i}}\right) .
$$

If we define the linear operator $L(H)$ as

$$
L(H) f \equiv\{f, H\},
$$

then we can write the formal solution of Hamilton's equations,

$$
f(t+\Delta t)=\exp [\Delta t L(H)] f(t) .
$$

In general the operator $\exp [\Delta t L(H)]$ cannot be expressed exactly in a simple form. Therefore we approximate 
$\exp [\Delta t L(H)]$ with an operator correct up to a certain order in $\Delta t$. Let us write $L(H)$ as

$$
\begin{aligned}
L(H) & =L\left(\frac{1}{2} p^{2}\right)+L[S(q)] \\
& =T+V,
\end{aligned}
$$

where $T \equiv L\left(\frac{1}{2} p^{2}\right)$ and $V \equiv L[S(q)]$. The 2LF integrator is given by decomposing $e^{\Delta t(T+V)}$ as

$$
\exp [\Delta t(T+V)]=\exp \left(\frac{1}{2} \Delta t T\right) \exp (\Delta t V) \exp \left(\frac{1}{2} \Delta t T\right)+\mathcal{O}\left(\Delta t^{3}\right) .
$$

We call $G_{2}(\Delta t)$ the 2LF integrator,

$$
G_{2}(\Delta t) \equiv \exp \left(\frac{1}{2} \Delta t T\right) \exp (\Delta t V) \exp \left(\frac{1}{2} \Delta t T\right) .
$$

The integrator $G_{2}(\Delta t)$ amounts to mapping $q$ and $p$ to new variables as

$$
\begin{aligned}
& \left(\begin{array}{l}
q(t+\Delta t) \\
p(t+\Delta t)
\end{array}\right)=\left(\begin{array}{cc}
1 & \frac{1}{2} \Delta t \\
0 & 1
\end{array}\right)\left(\begin{array}{cc}
1 & 0 \\
-\frac{\partial S(q)}{\partial q} \frac{\Delta t}{q} & 1
\end{array}\right)\left(\begin{array}{cc}
1 & \frac{1}{2} \Delta t \\
0 & 1
\end{array}\right)\left(\begin{array}{l}
q(t) \\
p(t)
\end{array}\right) \\
& \equiv G_{2}(\Delta t)\left(\begin{array}{l}
q(t) \\
p(t)
\end{array}\right) .
\end{aligned}
$$

This map is symplectic. This is easy to see, since the three matrices representing the elementary substeps are triangular with determinant 1 . It is also exactly time reversible, $G_{2}(\Delta t) G_{2}(-\Delta t)=1$.

An equivalent algorithm is obtained by interchanging $T$ and $V$ in Eq. (9).

Higher order integrators can also be found by decomposing $e^{\Delta t(T+V)}$ to the desired order. Although the decomposition to a higher order is a nontrivial problem with no unique solution, there is a simple recursive construction scheme which generates higher order integrators from lower order ones $[3,10,8]$. In this scheme, the $(2 k+2)$ th order integrator is given by

$$
G_{2 k+2}(\Delta t)=G_{2 k}\left(b_{1} \Delta t\right) G_{2 k}\left(b_{2} \Delta t\right) G_{2 k}\left(b_{1} \Delta t\right),
$$

where

$$
\begin{gathered}
b_{1}=\frac{1}{2-2^{1 /(2 k+1)}}, \\
b_{2}=1-2 b_{1}=-\frac{2^{1 /(2 k+1)}}{2-2^{1 /(2 k+1)}} .
\end{gathered}
$$

Let us call the integrators of Eq. (12) recursive construction (RC) integrators. These integrators are symplectic and constructed in a symmetric way, thus time reversible, i.e., $G_{2 k+2}(\Delta t) G_{2 k+2}(-\Delta t)=1$. Note that $b_{2}$ is negative. The appearance of negative coefficients in higher order integrators is inevitable: beyond the second-order decomposition there is no decomposition scheme having positive coefficients only [11]. However if we include the commutator $[V,[T, V]]$ in the decomposition we may circumvent this situation $[12,13]$, and integrators with all positive coefficients can be constructed. Even so, the inclusion of this commutator requires the calculation of the gradient of the force, which increases the computational cost. If the force-gradient calculations are computationally simple for the system considered, then it would be worth considering such integrators. For lattice QCD, it is unclear that such force-gradient integrators have advantages over the non-force-gradient ones. We do not consider such force-gradient integrators here.

\section{B. Minimum norm construction scheme}

Although the recursive construction scheme makes it easy to construct higher order integrators to any order, their performance may not be optimal, since the number of force calculations grows rapidly with the order of the integrator. More generally, one can decompose $e^{\Delta t(T+V)}$ as

$$
\exp (\Delta t(T+V))=\Pi_{i}^{k} \exp \left(c_{i} \Delta t T\right) \exp \left(d_{i} \Delta t V\right)+\mathcal{O}\left(\Delta t^{n+1}\right),
$$

where $\sum_{i}^{k} c_{i}=\sum_{i}^{k} d_{i}=1$. Moreover, in order to form a timereversible integrator certain relations must hold. For instance if we take $k=3$, the following equations must be satisfied: (i) $c_{1}=c_{4}, c_{2}=c_{3}, d_{1}=d_{3}, d_{4}=0$ or (ii) $c_{1}=0, c_{2}=c_{4}, d_{1}=d_{4}, d_{2}$ $=d_{3}$. For time-reversible integrators, the error terms with odd $n$ always vanish $[3,8,11]$. Thus time-reversible integrators have a leading error term $\mathcal{O}\left(\Delta t^{n+1}\right)$ with $n$ even.

The error term $\mathcal{O}\left(\Delta t^{n+1}\right)$ consists of commutators of $T$ and $V$. For instance, the leading error terms of the second- and fourth-order integrators are, respectively [6],

$$
\mathcal{O}\left(\Delta t^{3}\right)=\alpha[T,[V, T]]+\beta[V,[V, T]],
$$

and

$$
\begin{aligned}
\mathcal{O}\left(\Delta t^{5}\right)= & \gamma_{1}[T,[T,[T,[T, V]]]]+\gamma_{2}[T,[T,[V,[T, V]]]] \\
& +\gamma_{3}[V,[T,[T,[T, V]]]]+\gamma_{4}[V,[V,[T,[T, V]]]],
\end{aligned}
$$

where $\alpha, \beta$ and $\gamma_{i}$ depend on $c_{i}$ and $d_{i}$.

One strategy to find optimal integrators in the absence of further information about the operators $T$ and $V$ is to minimize the norm of the error coefficients. For the case of Eqs. (16) and (17), this strategy implies minimizing the following error functions:

$$
\mathcal{E}_{3} \equiv \sqrt{\alpha^{2}+\beta^{2}}
$$

and

$$
\mathcal{E}_{5} \equiv \sqrt{\gamma_{1}^{2}+\gamma_{2}^{2}+\gamma_{3}^{2}+\gamma_{4}^{2}} .
$$

Omelyan et al. [6] found a class of integrators by following this strategy. ${ }^{1}$ Among the new integrators which they identified, they found several "outstanding" integrators having especially small norms of the error coefficients. In this analy-

\footnotetext{
${ }^{1}$ Note that in some cases, the set of polynomial equations defining the optimal decomposition can be solved analytically, even beyond the second-order case [14].
} 
sis, we consider the new second- and fourth-order integrators which they recommend as outstanding integrators, and which are described as follows.

\section{Second-order minimum norm $(2 M N)$ integrator}

Omelyan et al. $[6,15]$ obtained the following new secondorder integrator:

$$
I_{2 \mathrm{MN}}(\Delta t)=e^{\lambda \Delta t T} e^{(\Delta t / 2) V} e^{(1-2 \lambda) \Delta t T} e^{(\Delta t / 2) V} e^{\lambda \Delta t T},
$$

where $\lambda$ takes value $\lambda_{c}$,

$$
\begin{aligned}
\lambda_{c} & =\frac{1}{2}-\frac{(2 \sqrt{326}+36)^{1 / 3}}{12}+\frac{1}{(6 \sqrt{326}+36)^{1 / 3}} \\
& \approx 0.1931833275037836 .
\end{aligned}
$$

This value of $\lambda$ minimizes $\sqrt{\alpha(\lambda)^{2}+\beta(\lambda)^{2}}$, where

$$
\begin{gathered}
\alpha(\lambda)=\frac{1-6 \lambda+6 \lambda^{2}}{12}, \\
\beta(\lambda)=\frac{1-6 \lambda}{24}
\end{gathered}
$$

as can be derived from the expansion of (20).

This integrator requires two force calculations per step. Thus, compared to the 2LF integrator, it has twice the computational cost. The norm of the error coefficients $\mathcal{E}_{3}$, however, is a factor of 10 smaller $\left(\mathcal{E}_{3}^{2 \mathrm{LF}} / \mathcal{E}_{3}^{2 \mathrm{MN}} \approx 10.9\right.$ [15]). As we will see later, the error of a second-order integrator at the end of a hybrid Monte Carlo trajectory is expected to be proportional to $\Delta t^{2}$. Therefore, even after taking into account the increased computational cost, we expect that the $2 \mathrm{MN}$ integrator will perform better than the 2LF integrator, by a factor $\approx \sqrt{10.9} / 2$. We will numerically confirm this in the next section, and later we will further try to tune the integrator by modifying the error function.

\section{Fourth-order minimum norm (4MN) integrator}

At the beginning of the MD integration one can start the integration with either $q$ or $p$. Usually we do not consider this freedom seriously since for the second-order integrator the choice of the starting variable does not make a significant difference in performance. ${ }^{2}$ In general, however, the performance could be different depending on the choice of the starting variable. In fact, the optimal integrator itself could also be different depending on the starting variable.

This is precisely what Omelyan et al. found for higher order MN integrators. Let us call velocity version ${ }^{3}$ the integrator starting by integrating $p$ and position version the integrator starting by integrating $q$. For the optimal fourth-order MN integrators with five force calculations they found that the velocity version has smaller errors than the position version. Actually we have tested both integrators and also found

\footnotetext{
${ }^{2}$ Actually there is a small difference, which we identify later.

${ }^{3}$ One could say momentum version. Here we follow the convention in the literature.
}

that typically the error of the velocity version is a few times smaller than that of the position version. In the following numerical tests we use only the velocity version of the fourth-order $\mathrm{MN}$ integrators with five force calculations (4MN5FV) which is written as [6]

$$
\begin{aligned}
I_{4 \mathrm{MN} 5 \mathrm{FV}}(\Delta t)= & e^{\theta \Delta t V} e^{\rho \Delta t T} e^{\lambda \Delta t V} e^{\mu \Delta t T} e^{[1-2(\lambda+\theta)](\Delta t / 2) V} \\
& \times e^{[1-2(\mu+\rho)] \Delta t T} e^{[1-2(\lambda+\theta)](\Delta t / 2) V} \\
& \times e^{\mu \Delta t T} e^{\lambda \Delta t V} e^{\rho \Delta t T} e^{\theta \Delta t V},
\end{aligned}
$$

where

$$
\begin{gathered}
\theta=0.08398315262876693, \\
\rho=0.2539785108410595, \\
\lambda=0.6822365335719091, \\
\mu=-0.03230286765269967 .
\end{gathered}
$$

Furthermore we have also tested the position version of the fourth-order MN integrators with four force calculations (4MN4FP) given by [6]

$$
\begin{aligned}
I_{4 \mathrm{MN} 4 \mathrm{FP}}(\Delta t)= & e^{\rho \Delta t T} e^{\lambda \Delta t V} e^{\theta \Delta t T} e^{(1-2 \lambda)(\Delta t / 2) V} e^{[1-2(\theta+\rho)] \Delta t T} \\
& \times e^{(1-2 \lambda)(\Delta t / 2) V} e^{\theta \Delta t T} e^{\lambda \Delta t V} e^{\rho \Delta t T}
\end{aligned}
$$

where

$$
\begin{gathered}
\rho=0.1786178958448091, \\
\theta=-0.06626458266981843, \\
\lambda=0.7123418310626056 .
\end{gathered}
$$

The velocity version (4MN4FV) is expected to have a similar error to the position version 4MN4FP above [6]. Thus we used the 4MN4FP integrator which has one less force evaluation per MD trajectory.

\section{NUMERICAL TESTS OF THE NEW INTEGRATORS}

\section{A. Lattice QCD action}

We use the plaquette Wilson gauge and standard Wilson fermion actions with two degenerate fermion flavors [16]. The partition function is given by

$$
Z=\int \mathcal{D} U \operatorname{det}\left[M(U) M(U)^{\dagger}\right] \exp \left[-S_{g}(U)\right],
$$

with $S_{g}(U)$ the gauge action given by

$$
S_{g}(U)=\frac{\beta}{3} \sum_{U_{p}} \operatorname{Tr}\left[1-U_{p}(U)\right],
$$

where $U_{p}(U)$ stands for the plaquette, $\beta$ is the gauge coupling and $U$ is an $\mathrm{SU}(3)$ link variable. $M(U)$ is the Wilson Dirac operator defined by 


$$
\begin{aligned}
M_{i j}(U)= & \delta_{i, j}+\kappa \sum_{\mu}\left[\left(\gamma_{\mu}-1\right) U_{i, \mu} \delta_{i, j+\mu}\right. \\
& \left.-\left(\gamma_{\mu}+1\right) U_{i-\mu, \mu}^{\dagger} \delta_{i, j+\mu}\right],
\end{aligned}
$$

where $\kappa$ is the hopping parameter and $\gamma_{\mu}$ are the $\gamma$ matrices. The inverse of the hopping parameter $\kappa$ is related to the quark mass $m$ and the value $\kappa$ where $m$ is zero is denoted by $\kappa_{c}$. As $m$ decreases the eigenvalues of the matrix $M$ become small and at the zero quark mass limit the matrix $M$ becomes singular.

Using pseudofermion fields $\phi$ the partition function is reexpressed as

$$
Z=\int \mathcal{D} U \mathcal{D} \phi^{\dagger} \mathcal{D} \phi \exp \left[-\phi^{\dagger}\left(M(U) M(U)^{\dagger}\right)^{-1} \phi-S_{g}(U)\right] .
$$

Furthermore introducing momenta $p$, we obtain

$$
Z=\int \mathcal{D} U \mathcal{D} \phi^{\dagger} \mathcal{D} \phi \mathcal{D} p \exp \left[-H\left(U, \phi, \phi^{\dagger}, p\right)\right],
$$

where

$$
H\left(U, \phi, \phi^{\dagger}, p\right)=\frac{1}{2} \sum p^{2}+\phi^{\dagger}\left(M(U) M(U)^{\dagger}\right)^{-1} \phi+S_{g}(U)
$$

is the Hamiltonian we consider in our numerical tests.

\section{B. Hybrid Monte Carlo algorithm}

The HMC algorithm combines MD and Metropolis accept/reject steps [1] to form a Markov chain. Starting from an "old" configuration $\{U\}$, a "candidate" configuration $\left\{U^{\prime}\right\}$ is obtained by (i) drawing momenta $\{p\}$ and pseudofermion fields $\{\phi\}$ from Gaussian distributions $\exp \left(-\frac{1}{2} p^{2}\right)$ and $\exp \left[-\phi^{\dagger}\left(M(U) M(U)^{\dagger}\right)^{-1} \phi\right]$, respectively; (ii) integrating Hamilton's equations of motion Eq. (2) with a discrete stepsize integrator. In order to maintain detailed balance the integrator in the MD step must satisfy two conditions: simplecticity and time reversibility. Let $T_{\mathrm{MD}}(\Delta t)$ be an elementary MD step with a discrete step size $\Delta t$. $T_{\mathrm{MD}}(\Delta t)$ evolves $(p, q)$ to $\left(p^{\prime}, q^{\prime}\right)$,

$$
T_{\mathrm{MD}}(\Delta t):(p, q) \rightarrow\left(p^{\prime}, q^{\prime}\right) .
$$

The time reversibility condition means the following is satisfied:

$$
T_{\mathrm{MD}}(-\Delta t):\left(p^{\prime}, q^{\prime}\right) \rightarrow(p, q) .
$$

The simplecticity is the condition that the phase space volume is conserved, $d p d q=d p^{\prime} d q^{\prime}$. The symplectic integrators in Sec. II satisfy the above two conditions.

This elementary MD step is performed repeatedly to integrate the equations up to a certain "length" of the MD trajectory. Here the trajectory length is set to unity. In general the integrator cannot solve the Hamilton's equations of motion exactly and thus the energy is not conserved. Let $\Delta H$ be the change in energy caused by the integrator at the end of the trajectory. This error is corrected by the Metropolis test, which accepts the candidate configuration $\left\{U^{\prime}\right\}$ at the end of the trajectory with probability $p=\min [\exp (-\Delta H), 1]$. If the candidate configuration is rejected, the old configuration $\{U\}$ is included again in the Markov chain.

Thus, a trade-off must be achieved between two conflicting goals: good energy conservation, because it ensures high acceptance probability in the Metropolis step, and low computer cost. This is why the choice of integrator plays a crucial role.

The fermionic part of the force $\partial H / \partial U$ is given by

$$
\frac{\partial H_{\text {fermionic }}}{\partial U}=-\phi^{\dagger}\left(M M^{\dagger}\right)^{-1} \frac{\partial\left(M M^{\dagger}\right)}{\partial U}\left(M M^{\dagger}\right)^{-1} \phi .
$$

Since the matrix $M$ is singular at the quark mass $m=0$, as $m$ decreases the fermionic force diverges. Thus HMC simulations in lattice QCD become unstable at small quark masses, unless the stepsize is reduced in proportion to $m$.

\section{Error of Hamiltonian and acceptance}

Here we summarize the expected behavior for the error of the Hamiltonian and the acceptance of the HMC algorithm. The $n$th order integrator causes $\mathcal{O}\left(\Delta t^{n+1}\right)$ integration errors for $q$ and $p$ after one step. However the error in the Hamiltonian at the end of a unit-time trajectory ${ }^{4}$ is $\mathcal{O}\left(\Delta t^{n}\right)$. Thus $\Delta H \sim \Delta t^{n}$. Furthermore from Creutz's equality $\langle\exp (\Delta H)\rangle$ $=1[18]$ we expect

$$
\langle\Delta H\rangle \sim\left\langle\Delta H^{2}\right\rangle \propto V \Delta t^{2 n},
$$

where $V$ is the volume of the system. Thus the root mean square of the error of the Hamiltonian at small $\Delta t$ is expected to be

$$
\left\langle\Delta H^{2}\right\rangle^{1 / 2} \approx C_{n} V^{1 / 2} \Delta t^{n},
$$

where $C_{n}$ is a Hamiltonian- and integrator-dependent coefficient. Using $\left\langle\Delta H^{2}\right\rangle^{1 / 2}$, the acceptance of the HMC algorithm for large volumes is given by [17]

$$
\left\langle P_{\text {acc }}\right\rangle=\operatorname{erfc}\left(\left\langle\frac{1}{8} \Delta H^{2}\right\rangle^{1 / 2}\right) .
$$

For small $\left\langle\Delta H^{2}\right\rangle^{1 / 2}$, one may use the approximate formula,

$$
\left\langle P_{\text {acc }}\right\rangle=\exp \left(-\frac{1}{\sqrt{2 \pi}}\left\langle\Delta H^{2}\right\rangle^{1 / 2}\right),
$$

which is applicable for $P_{\text {acc }} \geqslant 20 \%$ [4]. The performance of integrators can be measured by the (inverse of the) work per accepted trajectory, i.e., by the product of the acceptance and step size, $P_{\text {acc }} \times \Delta t$. The best performance of integrators is obtained at the step size which maximize $P_{\text {acc }} \times \Delta t$. Using Eqs. (38) and (40) we obtain the optimal acceptance which maximizes $P_{\text {acc }} \times \Delta t$ as [4]

\footnotetext{
${ }^{4} \Delta H$ does not increase linearly with trajectory length. It increases linearly up to a certain characteristic length $l_{c}$ provided that $\Delta t$ is not too large, then saturates. Thus the accumulated error in the Hamiltonian is expected to be $\Delta H \sim \mathcal{O}\left(\Delta t^{n+1}\right) \times\left(l_{c} / \Delta t\right) \sim \mathcal{O}\left(\Delta t^{n}\right)$ [17].
} 


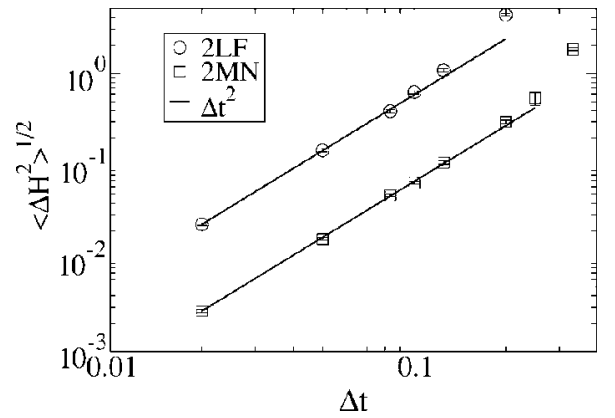

FIG. 1. $\left\langle\Delta H^{2}\right\rangle^{1 / 2}$ as a function of $\Delta t$. Simulations are performed at $\beta=5.00$ and $\kappa=0.160$ on $4^{4}$ lattices. The line proportional to $\Delta t^{2}$ is drawn to guide the eye.

$$
P_{\text {opt }}=\exp \left(-\frac{1}{n}\right),
$$

which does not depend on the details of the Hamiltonian but only on the order of the integrator. This result indicates that the optimal acceptance for any second-order integrator is about $61 \%$ which is consistent with the numerical results of $60 \%-70 \%$ [4]. Equation (41) also indicates that the optimal acceptance increases with the order of the integrator, $78 \%$ for fourth order and $85 \%$ for sixth order.

\section{Performance of second-order MN (2MN) integrator}

Here we compare the efficiency of the new 2MN integrator with that of the $2 \mathrm{LF}$ integrator. For second-order integrators, from Eq. (38) $\left\langle\Delta H^{2}\right\rangle^{1 / 2}$ at small $\Delta t$ is expected to be $C_{2} V^{1 / 2} \Delta t^{2}$. We measure the coefficient $C_{2}$ for both integrators at small enough $\Delta t$, and by comparing the coefficients we obtain the performance of the $2 \mathrm{MN}$ integrator relative to the 2LF integrator.

Figure 1 shows $\left\langle\Delta H^{2}\right\rangle^{1 / 2}$ as a function of step size $\Delta t$ at $\beta=5.00$ and $\kappa=0.160$ on $4^{4}$ lattices. We see that $\left\langle\Delta H^{2}\right\rangle^{1 / 2}$ is proportional to $\Delta t^{2}$ as expected and the error of the $2 \mathrm{MN}$ integrator is about 10 times smaller than that of the standard 2LF integrator at any $\Delta t$ until instabilities show up.

Figure 2 shows the ratio $C_{2 \mathrm{LF}} / C_{2 \mathrm{MN}}$ as a function of $\kappa$. The coefficients $C_{2 \mathrm{LF}}$ and $C_{2 \mathrm{MN}}$ are extracted by using Eq. (38) for the second-order with simulations at a small value of

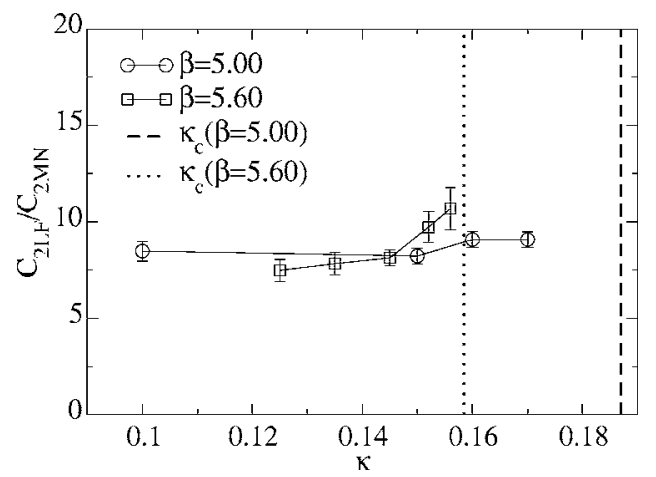

FIG. 2. $C_{2 \mathrm{LF}} / C_{2 \mathrm{MN}}$ as a function of $\kappa$. Simulations at $\beta$ $=5.00(5.60)$ are performed on $4^{4}\left(8^{4}\right)$ lattices.
$\Delta t$. As seen in the figure, $C_{2 \mathrm{LF}} / C_{2 \mathrm{MN}}$ is about 10 , which means that the error of the $2 \mathrm{MN}$ integrator is about 10 times smaller than that of the $2 \mathrm{LF}$ integrator. This is consistent with the theoretical expectation of Omelyan et al. If we take $C_{2 \mathrm{LF}} / C_{2 \mathrm{MN}} \approx 10$, this means that the step size of the $2 \mathrm{MN}$ integrator can be increased by a factor $3(\approx \sqrt{10})$ over that of the $2 \mathrm{LF}$ integrator, as long as the error still behaves as $\Delta t^{2}$. Since the $2 \mathrm{MN}$ integrator has two force calculations per elementary step, the efficiency should be measured by $\sqrt{C_{2 \mathrm{LF}} / C_{2 \mathrm{MN}}} / 2$, which is about 1.5 . Thus it is concluded that the $2 \mathrm{MN}$ integrator is about $50 \%$ faster than the $2 \mathrm{LF}$ integrator.

Of course, this assessment rests on the assumption that the step size can indeed be increased without running into instabilities, so that the limiting factor in the step size comes from the error accumulation. Note that the $2 \mathrm{MN}$ integrator appears no worse, or perhaps slightly better, than the $2 \mathrm{LF}$ with respect to instabilities: departure from the quadratic behaviour $\left\langle\Delta H^{2}\right\rangle^{1 / 2} \propto \Delta t^{2}$ starts at similar values of $\left\langle\Delta H^{2}\right\rangle^{1 / 2}$ in Fig. 1, and appears more gradual.

\section{E. Comparison of second- and fourth-order MN integrators}

The efficiency of higher order integrators should be measured against lower order ones. From the above analysis we know that the $2 \mathrm{MN}$ integrator is more efficient than the $2 \mathrm{LF}$ integrator. Therefore we compare the $4 \mathrm{MN}$ integrator with the $2 \mathrm{MN}$ integrator. Assuming Eq. (38) the comparison could be done by following the analysis of Ref. [4]. However we found a problem with the $4 \mathrm{MN}$ integrator. Namely the error of the Hamiltonian $\left\langle\Delta H^{2}\right\rangle^{1 / 2}$ is not simply described by Eq. (38) but is dominated by higher order terms in $\Delta t$ already at small $\left\langle\Delta H^{2}\right\rangle^{1 / 2}$. Figure 3 shows $\left\langle\Delta H^{2}\right\rangle^{1 / 2}$ on $8^{4}$ lattices as a function of $\Delta t$. As seen in the figure (top), at a fixed step size the error of the 4MN5FV integrator is about 1000 times smaller than the previously known fourth-order integrator (4RC), which is consistent with the theoretical expectation [6]. The expected behavior of $\left\langle\Delta H^{2}\right\rangle^{1 / 2} \approx C_{4} V^{1 / 2} \Delta t^{4}$, however, is seen only at small $\left\langle\Delta H^{2}\right\rangle^{1 / 2}$. We are only interested in the region of $0.1 \leqslant\left\langle\Delta H^{2}\right\rangle^{1 / 2} \leqslant 1$ which corresponds to an acceptance of $60 \%-95 \% .^{5}$ In this region, $\left\langle\Delta H^{2}\right\rangle^{1 / 2}$ of the $4 \mathrm{MN} 5 \mathrm{FV}$ integrator is dominated by higher order terms in $\Delta t$, which results in that $\left\langle\Delta H^{2}\right\rangle^{1 / 2}$ grows rapidly with $\Delta t$. This observation makes the $4 \mathrm{MN}$ integrator unattractive on practical lattice sizes.

Although the 4MN4FP integrator seems to be more stable than the $4 \mathrm{MN} 5 \mathrm{FV}$ integrator, it also shows the deviation from the $\Delta t^{4}$ line at small $\left\langle\Delta H^{2}\right\rangle^{1 / 2}$ (see Fig. 3). Thus compared to the $2 \mathrm{MN}$ integrator, the $4 \mathrm{MN}$ integrators tested here seem unattractive. As the quark mass $m$ decreases, it is often seen that the integrator becomes unstable [20], because the force increases as $1 / \mathrm{m}$. In lattice QCD calculations the parameter region of small quark masses is the physically interesting one. In this region the $4 \mathrm{MN}$ integrators may easily show instability, which limits their applicability.

\footnotetext{
${ }^{5}$ Figure 1 of Ref. [4].
} 

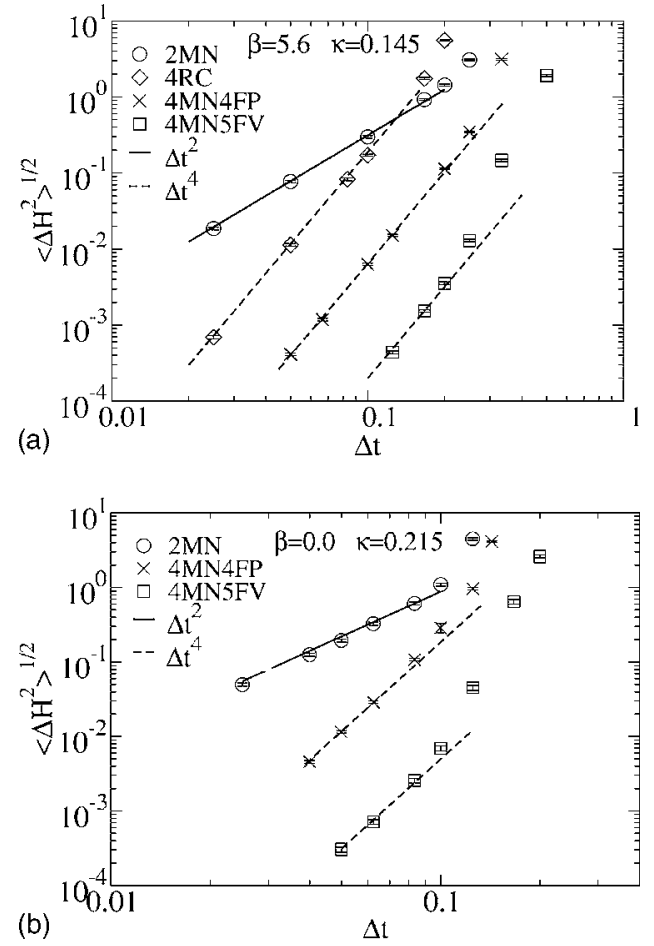

FIG. 3. $\left\langle\Delta H^{2}\right\rangle^{1 / 2}$ as a function of $\Delta t$. Simulations are performed on $8^{4}$ lattices. 4RC stands for the fourth-order integrator obtained by Eq. (12). The lines proportional to $\Delta t^{n}$ are drawn to guide the eye.

At finite temperature, however, the coefficients $C_{n}$ behave differently from those at zero temperature. Typically we expect $C_{n}^{T \neq 0} \leqslant C_{n}^{T=0}$. Therefore at finite temperature we may be lead to a different conclusion and this must be studied numerically. A numerical test showed that at finite temperature the $4 \mathrm{RC}$ integrator performs better than the $2 \mathrm{LF}$ integrator on lattices larger than a minimum size [5]. We have made the same test for the $2 \mathrm{MN}$ and $4 \mathrm{MN} 5 \mathrm{FV}$ integrators on an $18^{3}$ $\times 4$ lattice at $\beta=5.75$ and $\kappa=0.1525$. For the $2 \mathrm{MN}$ integrator the acceptance is measured to be about 0.6 at $\Delta t=0.1$ and for the $4 \mathrm{MN} 5 \mathrm{FV}$ integrator the acceptance is about 0.8 at $\Delta t$ $=0.37$. These values of the acceptance are close enough to the optimal acceptance given by Eq. (41). The gain of the $4 \mathrm{MN} 5 \mathrm{FV}$ integrator over the $2 \mathrm{MN}$ one is calculated by

$$
G=\frac{\left(P_{\mathrm{acc}} \times \Delta t\right)_{4 \mathrm{th}}}{\kappa_{42}\left(P_{\mathrm{acc}} \times \Delta t\right)_{2 \mathrm{nd}}},
$$

where $\kappa_{42}$ is the relative cost factor and $\kappa_{42}=2.5$ for the $2 \mathrm{MN}$ and $4 \mathrm{MN} 5 \mathrm{FV}$ integrators. Substituting the measured values into $G, G$ is calculated to be $\approx 2$, which shows that the $4 \mathrm{MN} 5 \mathrm{FV}$ integrator is more effective. Thus at finite temperature there is room to use a $4 \mathrm{MN}$ integrator depending on the simulation parameters.

\section{TUNING THE 2MN INTEGRATOR}

The strategy to minimize Eqs. (18) and (19) is based on the assumption that the errors coming from the two commutators $[T,[V, T]]$ and $[V,[V, T]]$ are equally dominant. In
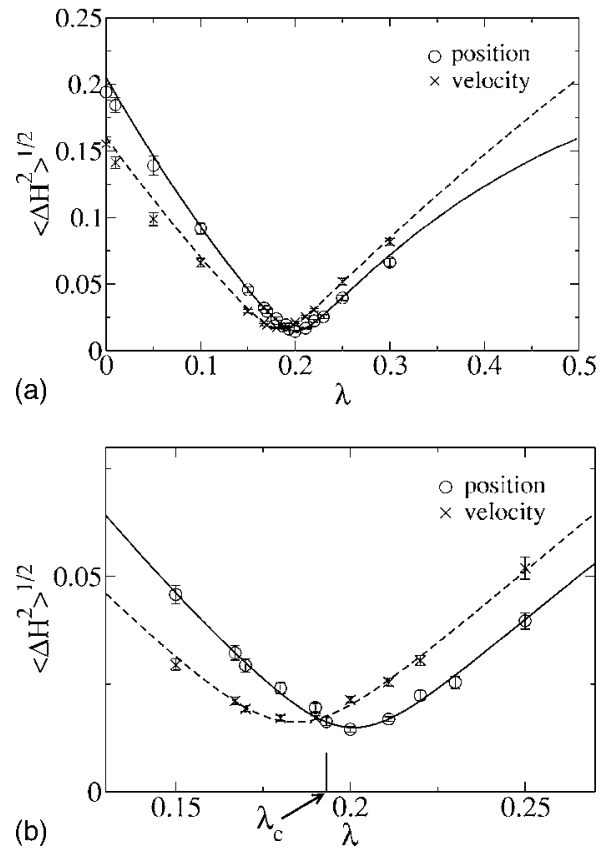

FIG. 4. $\left\langle\Delta H^{2}\right\rangle^{1 / 2}$ as a function of $\lambda$. The bottom figure is a zoom of the top figure. Simulations are performed at $\beta=5.00$ and $\kappa$ $=0.160$ on $4^{4}$ lattices with $\Delta t=0.05$. The lines are determined from simulations of the position version integrator at $\lambda_{1}$ and $\lambda_{2}$. The position version has a small advantage over the velocity version, since it gives a slightly reduced minimum RMS error (bottom).

general this simplifying assumption does not hold. Here we try to minimize a more general form of the error function. Let us assume the following form of $\left\langle\Delta H^{2}\right\rangle^{1 / 2}$ :

$$
\begin{aligned}
\left\langle\Delta H^{2}\right\rangle^{1 / 2} & \approx \sqrt{\alpha(\lambda)^{2} f(\Delta t)^{2}+\beta(\lambda)^{2} g(\Delta t)^{2}} \\
& =\sqrt{\alpha(\lambda)^{2} f^{2}+\beta(\lambda)^{2} g^{2}} \Delta t^{2},
\end{aligned}
$$

where $\alpha(\lambda)$ and $\beta(\lambda)$ are given by Eqs. (22) and (23), and $f^{2}$ and $g^{2}$ are unknown parameters, to be determined from numerical simulations. In general, by performing simulations at two values of $\lambda$ one can determine $f^{2}$ and $g^{2}$ numerically. The determination can be made easier by noticing that $\alpha\left(\lambda_{1}\right)$ and $\beta\left(\lambda_{2}\right)$ are zero at $\lambda_{1}=(1-1 / \sqrt{3}) / 2$ and $\lambda_{2}=1 / 6$, respectively. By simulating at $\lambda_{1}$ and $\lambda_{2}$ we immediately obtain $f^{2}$ and $g^{2}$.

Figure 4 shows $\left\langle\Delta H^{2}\right\rangle^{1 / 2}$ at $\beta=5.00$ and $\kappa=0.160$ as a function of $\lambda$. We see that the optimal $\lambda$ at the minimum of $\left\langle\Delta H^{2}\right\rangle^{1 / 2}$ is slightly different from $\lambda_{c}$, the value of Eq. (21). Moreover the optimal $\lambda$ is different between the velocity and position version integrators. Here Eq. (20) is the position version integrator. The velocity version is obtained by interchanging $T$ and $V$ in Eq. (20). The lines in the figure are given by Eq. (44), with $f^{2}$ and $g^{2}$ determined by simulations of the position version of $2 \mathrm{MN}$ integrator at $\lambda_{1}$ and $\lambda_{2}$. To draw the dashed line for the velocity version, we simply interchange the values $f^{2}$ and $g^{2}$. Both lines describe the numerical results very well, down to $\lambda=0$. Note that the velocity version of $2 \mathrm{MN}$ integrator becomes the position version of $2 \mathrm{LF}$ integrator at $\lambda=0$, and vice versa. The position 


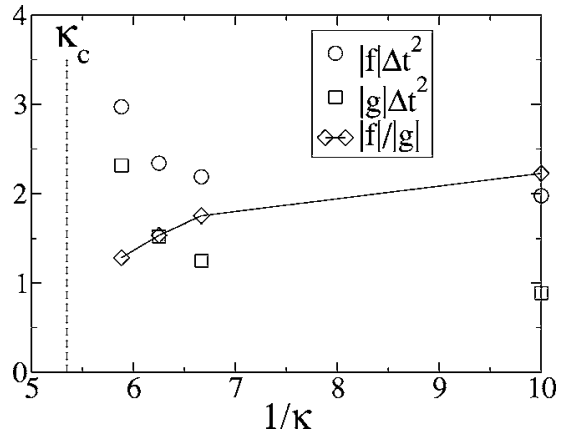

FIG. 5. $|f| \Delta t^{2},|g| \Delta t^{2}$, and $|f| /|g|$ as a function of $1 / \kappa$. Simulations are performed at $\beta=5.00$ on $4^{4}$ lattices with $\Delta t=0.05$. The dashed line indicates $\kappa_{c}=0.187$ [19].

version of the $2 \mathrm{MN}$ integrator gives a slightly smaller minimum. At $\lambda=0$ the velocity version of $2 \mathrm{MN}$ integrator has a smaller error than the position version, which means that the position version of $2 \mathrm{LF}$ integrator has a smaller error than the velocity version. This was already observed in Ref. [21]. Since the position version also leads to one less force evaluation by the end of a trajectory, we definitely recommend using the position version (for the $2 \mathrm{MN}$ and the $2 \mathrm{LF}$ integrators both): it requires less work and gives a higher acceptance.

The quality of our fit justifies a posteriori the ansatz made for the magnitude of the error Eq. (44). Indeed, to leading order $\Delta t^{4}$, the error $\left\langle\Delta H^{2}\right\rangle$ should be of the form

$$
\left\langle\Delta H^{2}\right\rangle=\left[\alpha(\lambda)^{2}\left\langle F^{2}\right\rangle+\alpha(\lambda) \beta(\lambda)\langle F G+G F\rangle+\beta(\lambda)^{2}\left\langle G^{2}\right\rangle\right] \Delta t^{4},
$$

where $F=[T,[V, T]]$ and $G=[V,[V, T]]$ from Eq. (16). We find that the crossterm $\langle F G\rangle$ is in our case "one order of magnitude smaller" than $\left\langle F^{2}\right\rangle$ and $\left\langle G^{2}\right\rangle$, indicating that the two operators are almost uncorrelated in our system. While this finding may not be true in general, it provides support for the minimum norm strategy of Omelyan et al. at least in the context of lattice QCD.

Figure 5 shows $|f| \Delta t^{2},|g| \Delta t^{2}$, and $|f| /|g|$ as a function of $1 / \kappa$ at $\beta=5.00$ on $4^{4}$ lattices $(\Delta t=0.05)$. As one approaches $\kappa_{c}$, both $|f|$ and $|g|$ increase. On the other hand, the ratio $|f| /|g|$ decreases. This is as expected since $g$ comes from the error term $[V,[V, T]]$ involving two factors of the fermion potential, versus one for $f$ and $[T,[V, T]]$. Although $|f| /|g|$ seems to approach one at $\kappa_{c}$, there is a possibility that it further goes down to zero and its limit must be carefully investigated. Note that when $|f| /|g|=1$, the optimal $\lambda$ becomes $\lambda_{c}$.

Figure 6 shows the optimal $\lambda$ as a function of $1 / \kappa$. We see that the optimal $\lambda$ is different from $\lambda_{c}$ and slightly larger.

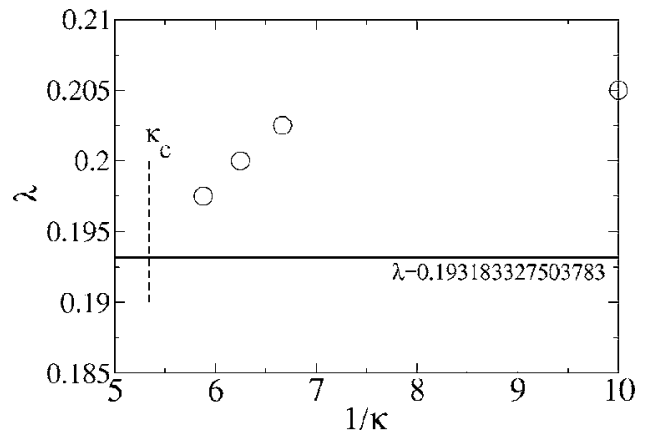

FIG. 6. Optimal $\lambda$ as a function of $1 / \kappa$. Simulations are performed at $\beta=5.00$ on $4^{4}$ lattices with $\Delta t=0.05$. The dashed line indicates $\kappa_{c}=0.187$.

\section{CONCLUSIONS}

We have tested the new second- and fourth-order integrators obtained by minimizing the norm of the error coefficients. We find that the $2 \mathrm{MN}$ integrator performs better than the conventional 2LF integrator, by about $50 \%$. Therefore we recommend to use the $2 \mathrm{MN}$ integrator in HMC simulations. Although in our tests we used the standard Wilson fermion action, the $2 \mathrm{MN}$ integrator can be used for any actions, e.g., KS fermions, improved actions, polynomial actions for odd flavors $[22,23]$. Moreover we may combine the $2 \mathrm{MN}$ integrator with other acceleration techniques such as multiple time step integration [9], multiple pseudofermions [24], and preconditioned actions [23].

The same $2 \mathrm{MN}$ integrator can also be used in the TrotterSuzuki decomposition of the partition function: $\exp (-\beta H)$ $=[\exp (-\Delta t H)]^{N}$, where $N=\beta / \Delta t$, in quantum Monte Carlo simulations, when a formulation in continuous imaginary time [25] is not practical.

Although at first sight, one can equivalently start by integrating over positions or velocities, we observe that integrating over positions first gives a slightly higher acceptance [21], with one less force evaluation at the end of a trajectory.

\section{ACKNOWLEDGMENTS}

The authors thank the Institute of Statistical Mathematics for the use of NEC SX-6, RCNP at Osaka University for the use of NEC SX-5 and the Yukawa Institute for the use of NEC SX-5, and Tony Kennedy for his comments on the paper. One of the authors (T.T.) would like to thank Professor Sigrist for hospitality during his stay at ETH Zürich. One of the authors (P.de F.) thanks the Kavli Institute for Theoretical Physics for hospitality during the completion of this paper. 
[1] S. Duane, A. D. Kennedy, B. J. Pendleton, and D. Roweth, Phys. Lett. B 195, 216 (1987).

[2] M. Campostrini and P. Rossi, Nucl. Phys. B 329, 753 (1990).

[3] M. Creutz and A. Gocksch, Phys. Rev. Lett. 63, 9 (1989).

[4] T. Takaishi, Comput. Phys. Commun. 133, 6 (2000).

[5] T. Takaishi, Phys. Lett. B 540, 159 (2002).

[6] I. P. Omelyan, I. M. Mryglod, and R. Folk, Comput. Phys. Commun. 151, 272 (2003).

[7] E. Forest and R. D. Ruth, Physica D 43, 105 (1990).

[8] H. Yoshida, Phys. Lett. A 150, 262 (1990).

[9] J. C. Sexton and D. H. Weingarten, Nucl. Phys. B 380, 665 (1992).

[10] M. Suzuki, Phys. Lett. A 146, 319 (1990).

[11] M. Suzuki, J. Math. Phys. 32, 400 (1991).

[12] M. Suzuki, Phys. Lett. A 201, 425 (1995).

[13] S. A. Chin, Phys. Lett. A 226, 344 (1997).

[14] A. D. Kennedy, "Monte Carlo algorithms and non-local actions," Fields Institute Communications (American Mathematical Society, Providence, RI, 2000), Vol. 26.
[15] I. P. Omelyan, I. M. Mryglod, and R. Folk, Phys. Rev. E 65, 056706 (2002).

[16] K. G. Wilson, Phys. Rev. D 10, 2445 (1974).

[17] S. Gupta, A. Irback, F. Karsch, and B. Petersson, Phys. Lett. B 242, 437 (1990).

[18] M. Creutz, Phys. Rev. D 38, 1228 (1988).

[19] A. Ukawa, Nucl. Phys. B, Proc. Suppl. 10, 66 (1989).

[20] See, e.g., A. D. Kennedy, Nucl. Phys. B, Proc. Suppl. 140, 190 (2005).

[21] R. Gupta, G. W. Kilcup, and S. R. Sharpe, Phys. Rev. D 38, 1278 (1988).

[22] T. Takaishi and P. de Forcrand, Int. J. Mod. Phys. C 13, 343 (2002).

[23] P. de Forcrand and T. Takaishi, Nucl. Phys. B, Proc. Suppl. 53, 968 (1997).

[24] M. Hasenbusch, Phys. Lett. B 519, 177 (2001).

[25] B. B. Beard and U. J. Wiese, Nucl. Phys. B, Proc. Suppl. 53, 838 (1997). 